\title{
Influência do posicionamento de barreiras rígidas centrais na visibilidade de projeto em rodovias de pista dupla
}

\author{
Érica Santos Matos ${ }^{1}$, Ana Paula Camargo Larocca², Felipe Issa Kabbach $\mathrm{Jr}^{3}$
}

\begin{abstract}
Stopping Sight Distance must be guaranteed throughout of a highway for safety reasons. However, cross section elements can become obstructions which interfere on design sight distances. This occurs when barriers are used instead of medians on bidirectional highways, especially along left curves. Therefore, this paper analyzes the barriers placement on hypothetical scenarios using three-dimensional graphics simulations. The lateral offset of the barrier in relation to the roadway was defined according to each design speed and each radius of the horizontal curve analysis. The diagrams created have potential use to definition of indicators for making decisions about the project, because the project was considered as a threedimensional model as a whole rather than analyzing separately the horizontal and vertical alignments and cross sections.
\end{abstract}

Keywords: Stopping Sight Distance. Barrier. Highway Geometric Design.

\begin{abstract}
Resumo: A distância de visibilidade de parada deve ser garantida ao longo de toda a extensão de uma rodovia por questões de segurança. Elementos da seção transversal podem se tornar obstruções que interferem nas distâncias de visibilidade do projeto. Este é o caso do uso de barreiras ao invés de canteiros centrais em rodovias bidirecionais, especialmente ao longo de curvas à esquerda. Desta forma, este trabalho analisa o posicionamento de barreiras em cenários hipotéticos com uso de simulações gráficas tridimensionais. O afastamento lateral da barreira, em relação à pista de rolamento, foi definido em função de cada velocidade de projeto e de raio de curva horizontal analisados. Os diagramas gerados têm potencial uso para definição de parâmetros indicadores na tomada de decisão do projeto viário, pois o projeto foi considerado como um modelo tridimensional como um todo ao invés de analisar separadamente os alinhamentos horizontal e vertical bem como as seções transversais.
\end{abstract}

Palavras-chave: Distância de Visibilidade de Parada. Barreira. Projeto Geométrico.

\section{INTRODUÇÃO}

O uso de barreiras rígidas como separadores físicos em rodovias bidirecionais é recorrente em estradas nacionais. Nas regiões serranas, por exemplo, a adoção destes separadores ao invés de canteiros centrais é uma solução para as limitações físicas da plataforma da via. O mesmo ocorre nos processos de duplicação, em que o espaço físico disponível para o desenvolvimento do projeto é restrito. A inserção de novos elementos - neste caso barreiras rígidas - em projetos existentes ou em desenvolvimento gera novas preocupações e cuidados nos quesitos de qualidade e segurança, principalmente no que tange às condições de visibilidade para o motorista. O motorista, quando dispõe de uma distância de visibilidade adequada, tem condições de captar as informações sobre a via e sobre o tráfego, interpretá-las, impor ao veículo

\footnotetext{
1 Escola Politécnica da Universidade de São Paulo Departamento de Engenharia de Transportes (erica_smatos@yahoo.com.br).

2 Escola de Engenharia de São Carlos da Universidade de São

Paulo -Departamento de Engenharia de Transportes

(larocca.ana@usp.br).

${ }^{3}$ Escola Politécnica da Universidade de São Paulo -

Departamento de Engenharia de Transportes

(fkabbach@planservi.com.br).

Manuscrito recebido em 28/08/2013 e aprovado para publicação em 08/05/2014. Este artigo é parte de TRANSPORTES v. 22, n. 2, 2014. ISSN: 2237-1346 (online). DOI: http://dx.doi.ora/10.14295/transportes.v22i2.723
}

trajetória e velocidade adequadas, tomar decisões a tempo e com segurança, além de ser capaz de imobilizar o veículo ou mesmo realizar outro tipo de manobra adequada quando surge algum obstáculo inesperado à sua frente (DER-SP, 2006). Sendo assim, para análises de visibilidade significativas, é necessário considerar a altura dos olhos do motorista e altura do objeto que represente uma situação de perigo conforme recomendações e manuais vigentes.

Todavia, o posicionamento de dispositivos de segurança deve atender às suas funções específicas, concomitantemente com a manutenção da visibilidade ao longo de toda a extensão da via. Logo, as interferências da composição geométrica de todos os elementos envolvidos devem ser analisadas e consideradas, visando conciliar funcionalidade com os padrões mínimos de visibilidade necessários. Fato é que a garantia de visibilidade ao trafegar em uma via têm relação direta na manutenção de padrões mínimos de segurança e dirigibilidade do motorista. Por isso, a visibilidade é tema recorrente na literatura, não sendo menos importante a conexão com o posicionamento de barreira sobre a via, onde diversos trabalhos apresentam análises da visibilidade disponível considerando o tipo de dispositivo de segurança, inclusive seu posicionamento na via, que é o foco do pre- 
sente trabalho. Hassan e Sarhan (2012), Arndt et al. (2010), Sanchez (1994) e Leisch (1989) são exemplos de trabalhos sobre este questionamento.

$\mathrm{O}$ conhecimento da interferência na visibilidade de dispositivos usualmente utilizados, como barreiras rígidas ou qualquer outra feição tridimensional, vêm subsidiar novas alternativas e auxiliar na tomada de decisões relacionadas ao projeto geométrico. Logo, o escopo do presente estudo foi investigar o posicionamento de barreiras rígidas para que não ocorram interferências na visibilidade necessária ao motorista durante o tráfego em determinada via, disponibilizando indicativos no quesito do posicionamento lateral da barreira e sua influência na Distância de Visibilidade de Parada (DVP).

\section{FUNDAMENTAÇÃO TEÓRICA}

\subsection{A visibilidade no projeto geométrico}

A visibilidade do motorista é um dos fatores mais importantes para a segurança e para a eficiência operacional de um veículo que trafega em qualquer via (Chou et al., 2010). O motorista precisa ter espaço livre suficiente para quando estiver em situação controversa (um obstáculo, por exemplo) ser capaz de imobilizar o veículo ou alterar sua trajetória, tendo consciência de sua ação. Além disso, o projeto viário deve atender a requisitos decorrentes das características do comportamento de parcela significativa dos motoristas e também do conjunto formado pelo veículo e pela pista (freios, suspensão, pneus, condições da superfície de rolamento), sobre determinadas condições (Pellegrini, 2006; DER-SP, 2006; DNER, 1999).

De tal forma que características do projeto geométrico são fatores determinantes na definição da condição de visibilidade existente (Ismail e Sayed, 2007). Esta é limitada pelas recorrentes mudanças de declividade e direção ao longo do desenvolvimento da via, com destaque para as curvas horizontais em trechos de corte e para as curvas verticais convexas. As curvas verticais côncavas apresentam restrições às condições de visibilidade da via no período noturno que, em geral, é definida como função da altura dos faróis dos veículos (Pellegrini, 2006; DER-SP, 2006).

No alinhamento horizontal, principalmente nos trechos de curvas, as restrições nas condições de visibilidade nem sempre resultam na fixação de padrões mínimos para o projeto geométrico, já que é possível minimizá-las - como, por exemplo, por meio do alargamento da plataforma de terraplena- gem, ou pelo abatimento do ângulo de inclinação de taludes em região de corte. Reduções de velocidade são verificadas para curvas horizontais com pequenos raios de curvatura (Pellegrini, 2006; DER-SP, 2006). Em contrapartida, neste trabalho pretende-se intervir na disposição dos elementos da seção transversal como solução.

Logo é necessário, sempre que possível, que o projeto geométrico de uma rodovia vise à maximização das distâncias de visibilidade do motorista, em prol de critérios de segurança, qualidade e conforto desde que exista espaço físico e viabilidade econômica para tal. Os parâmetros mensuráveis das condições de visibilidade considerados na elaboração de projetos geométricos de via se resumem à Distância de Visibilidade de Parada (DVP), à Distância de Visibilidade de Ultrapassagem (DVU) e à Distância de Visibilidade para Tomada de Decisão (DVTD). A primeira possui caráter obrigatório no qual o projeto deve garantir um valor mínimo a ser atendido em qualquer ponto da rodovia (AASHTO, 2011a) e é parâmetro importante no projeto, que interfere no projeto da rodovia, já que seus requisitos afetam todos os elementos geométricos envolvidos - alinhamentos horizontal e vertical, além da seção transversal (Neuman, 1989). As demais distâncias possuem valores recomendados que, quando atendidos, elevam o padrão técnico da via.

A formulação considerada neste trabalho para o cálculo da Distância de Visibilidade de Parada é a mesma adotada pelo Green Book da AASTHO (2011a) e pelo DER-SP (2006), em que:

$$
D V P=0,278 \cdot V \cdot t+\frac{V^{2}}{254 \cdot\left(\left(\frac{a}{9,81}\right)+i\right)}
$$

Onde

DVP: Distância de Visibilidade de Parada (m);

$V$ : Velocidade de projeto $(\mathrm{km} / \mathrm{h})$;

$t$ : Tempo de percepção e reação (s);

$a$ : Taxa de desaceleração $\left(\mathrm{m} / \mathrm{s}^{2}\right) ; \mathrm{e}$

$i$ : Declividade do greide $(\%)$.

Além de escolher um modelo para o cálculo da DVP, as análises de visibilidade precisam considerar o fato de que o motorista deve enxergar um objeto a uma dada distância necessária. Logo, sempre é necessário definir (a) uma distância mensurável - neste caso a DVP, (b) as características da posição do motorista e (c) características inerentes ao objeto que se pretende visualizar. 


\subsection{Barreira e a Visibilidade}

Por definição barreira é um dispositivo de segurança da via. Contudo este dispositivo pode ser empregado como separador físico de contenção central (ABNT, 2007), geralmente em casos onde não é possível a implantação de canteiros centrais devido a limitações da plataforma, em casos de duplicação de rodovias, ou ainda como delimitadores longitudinais.

Logo, a barreira pode inferir na garantia da DVP reduzindo a visibilidade disponível ao motorista, particularmente quando está situada no lado interno de uma curva (DNIT, 2010). AASHTO (2011b) apresenta várias considerações sobre o posicionamento de barreiras. O afastamento lateral, além do quesito visibilidade, deve ser imposto para que o motorista ao dirigir, não perceba a proximidade da barreira como um obstáculo e devido a isto, reduza a velocidade aplicada ou altere a trajetória do veículo. Os valores são relacionados com a velocidade de projeto e visam maior conforto e segurança no tráfego, além de auxiliar na manutenção de áreas limpas para a linha de visão do motorista. O termo apresentado para este afastamento lateral é linha de intimidação (do inglês shy line, segundo o Roadside Design Guide) ou linha de preocupação conforme a ABNT (NBR 15486:2007).

AASHTO (2011b) apresenta valores de afastamento lateral considerando o conceito de linha de intimidação (Tabela 1), cujo uso é sugerido quando a extensão longitudinal da barreira é curta, em pontos isolados da via, onde a percepção do objeto pelo motorista é prejudicada. Em grandes extensões, é comum no trecho inicial de implantação da barreira posicioná-la a uma distância maior da faixa de rolamento, impondo uma aproximação gradual auxiliando a percepção, não sendo crítico o não uso dos valores da Tabela 1 . Neste estudo, os valores sugeridos por AASHTO (2011b) não foram considerados para o estudo do afastamento lateral da barreira por causa de dois motivos: (1) apenas serão considerados casos onde a barreira é presente em grandes extensões da via e (2) tais valores não são aplicados expressivamente em âmbito nacional.

Leisch (1989) ressalta que o uso de barreiras de concreto em rodovias pode gerar inadequadas distâncias de visibilidade de parada disponíveis, principalmente em curvas e em áreas próximas a pontes e túneis. Para o autor, a solução é prover um afastamento variável entre faixa de rolamento e a barreira, quando posicionada na área central.
Tabela 1 - Afastamento Lateral segundo AASTHO (2011b)

\begin{tabular}{cc}
\hline Velocidade de Projeto & Afastamento \\
\hline $130 \mathrm{~km} / \mathrm{h}$ & $3,7 \mathrm{~m}$ \\
$120 \mathrm{~km} / \mathrm{h}$ & $3,2 \mathrm{~m}$ \\
$110 \mathrm{~km} / \mathrm{h}$ & $2,8 \mathrm{~m}$ \\
$100 \mathrm{~km} / \mathrm{h}$ & $2,4 \mathrm{~m}$ \\
$90 \mathrm{~km} / \mathrm{h}$ & $2,2 \mathrm{~m}$ \\
$80 \mathrm{~km} / \mathrm{h}$ & $2,0 \mathrm{~m}$ \\
$70 \mathrm{~km} / \mathrm{h}$ & $1,7 \mathrm{~m}$ \\
$60 \mathrm{~km} / \mathrm{h}$ & $1,4 \mathrm{~m}$ \\
$50 \mathrm{~km} / \mathrm{h}$ & $1,1 \mathrm{~m}$ \\
\hline
\end{tabular}

Sanchez (1994) realizou estudo sobre o posicionamento de barreiras e a visibilidade, por meio de simulações utilizando modelos tridimensionais. A quantidade de modelos utilizada no estudo foi limitada e o foco do autor estava em mensurar a DVP tridimensionalmente ao invés de utilizar os métodos bidimensionais tradicionais da época. $\mathrm{O}$ autor ainda realizou algumas ponderações sobre os afastamentos da barreira, considerando variações no perfil vertical, em casos de presença de curvas.

Arndt et al. (2010) avançam na investigação da relação entre o posicionamento de barreiras e visibilidade. Como solução, os autores apresentam um novo critério que consiste basicamente em utilizar os modelos de distâncias de visibilidade, com parâmetros menos conservadores, porém mais realistas. Algumas das alterações propostas são: o aumento da altura do objeto e da taxa de desaceleração, a redução da velocidade considerada, diminuição do tempo de percepção e reação em rodovias de alto padrão. Este estudo foi incorporado às normas australianas (AUSTROADS, 2010), como item específico que trata dos requisitos de distância de visibilidade em curvas horizontais quando na presença de barreiras, muros de contenção ou estruturas de pontes. Segundo AUSTROADS (2010) quando há visibilidade sobre a barreira, deve-se prover um afastamento lateral mínimo de 2,50 $\mathrm{m}$ até a faixa de rolamento interna da curva, quando analisado a DVP para veículo leve. Para veículos pesados, o afastamento mínimo é de 3,50 $\mathrm{m}$. Em casos onde não é possível a visibilidade, as normas sugerem o estudo de novos traçados horizontal e vertical de modo que o problema seja minimizado ou solucionado quando possível.

Em relação ao afastamento lateral de objetos ao longo de vias, Yang et al. (2012) constatou que os motoristas tendem a se mover longe do obstáculo (barreira) ou reduzir a velocidade temporariamente, por interpretar a barreira como fonte de 
risco à sua segurança. Rosey e Auberlet (2012) afirmam que as expectativas do motorista e a consistência geométrica são quesitos importantes em segurança rodoviária, pois inconsistências no projeto geométrico podem surpreender motoristas e levar a erros que aumentam o risco de acidente. Ainda segundo os autores, a variabilidade da posição do motorista lateralmente na pista pode ser um indicador de deficiência na geometria da via causado, por exemplo, pela posição da barreira. $\mathrm{Ou}$ seja, a solução é prover afastamento tal que o motorista sinta segurança ao dirigir, sem alterar sua trajetória, especialmente em condições atmosféricas e de visibilidade adversas (Yang et al., 2012; Chen et al., 2012).

Hassan e Sarhan (2012) investigaram os efeitos do alinhamento vertical nas distâncias de visibilidade de parada, com o foco no posicionamento de barreiras de concreto ao longo de curvas horizontais. A barreira utilizada no estudo tem altura de $0,81 \mathrm{~m}$. Em aplicativo gráfico desenvolvido pelos autores, foram gerados modelos tridimensionais de projeto genérico considerando a concordância do alinhamento horizontal e vertical, enquanto neste estudo, alterações foram impostas no modelo de modo a garantir a visibilidade ao longo da via. O aplicativo valida incremental e automaticamente afastamentos laterais para a análise de DVP.

Em seus resultados, os autores concluem que os valores fornecidos para a linha de intimação segundo o Roadside Design Guide (AASTHO, 2011b) provêm valores de distância de visibilidade inferiores à DVP necessária para a segurança do motorista. O estudo também confirmou que a DVP disponível em curvas com obstrução na forma de barreiras é dependente da coordenação com o alinhamento vertical. A sugestão dos autores é de expandir as análises da contribuição das características dos alinhamentos horizontal e vertical, propor novas modelagens de barreiras através de programas computacionais que permitam a análise tridimensional do projeto.

A forma da concepção do estudo de Hassan e Sarhan (2012) muito se assemelha ao empregado neste estudo, contudo a parametrização adotada foi distinta. Os autores enfatizaram os efeitos do alinhamento vertical, enquanto o presente trabalho prioriza as características das normativas nacionais vigentes com a adoção de declividades do greide constantes como simplificação à presença de curvas verticais.

\subsection{Visualização, Modelagem e Análise de Visibilidade}

Os projetos geométricos compreendem a elaboração de perfis horizontais, perfis verticais, perfis e seções transversais e longitudinais ao longo da extensão da obra viária. Devido a esta separação, há dificuldades em se visualizar o projeto integrado considerando suas peculiaridades técnicas e suas interferências sobre o meio. As técnicas de visualização tridimensional e modelagem começam a suprir tais dificuldades permitindo aos projetistas enxergarem o projeto final com objetividade.

Na Engenharia de Transportes, a visualização pode ser compreendida como a representação estática ou simulação de informações espaciais e geométricas das condições reais ou alterações propostas em vias de transportes e seus impactos associados sobre seu entorno como maneira suficiente para expressar ao projetista a realidade da geometria do projeto desenvolvido e ao leigo a real extensão das melhorias (NCHRP, 2006). Além disto, seu uso permite subsidiar decisões de alterações no traçado, visando garantir melhores qualidades técnica e estética ao projeto viário (Hixon III, 2007). O presente trabalho foi baseado em uma investigação gráfica - os resultados foram obtidos através da geometria do modelo e não por um equacionamento analítico.

Esta abordagem é encontrada em diversos trabalhos sobre análises de visibilidade, concordância de alinhamentos ou prospectos das aplicabilidades da visualização no desenvolvimento de projetos, como encontrado em Sanchez (1994); Janikula e Garrick (2002); Han, Middleton e Clayton (2006); Kuhn e Jha (2010); Chou et al. (2010); Hassan e Sarhan (2012).

O ganho ao se trabalhar com modelos é a compreensão do projeto como um todo, verificando como os elementos se relacionam entre si. $\mathrm{Na}$ análise de visibilidade, por sua vez, é possível verificar se a DVP é garantida ao longo de todo o trecho de análise, mensurando a amplitude de áreas sem obstrução ou ainda, quando for o caso, indicando áreas oclusas ao campo de visão do motorista.

A maior parte das normas e manuais vigentes indica que as análises de distâncias de visibilidade devem ser efetuadas em representações 2D, ou seja, através da verificação gráfica sobre os alinhamentos horizontal e vertical, separadamente (Ismail e Sayed, 2007). Hassan, Easa e Abd El Ha- 
lim (1997) compararam análises 2D e 3D e notaram que as distâncias de visibilidade disponíveis podem ser subestimadas ou superestimadas quando o método empregado é bidimensional. A tendência indicada por pesquisadores é expandir as análises em 3D (Chou et al., 2010), impulsionada pelo aprimoramento computacional necessário, tanto em hardware quanto em software.

Atualmente no mercado estão disponíveis programas que possuem módulos específicos para análise de visibilidade como o AutoCAD Civil $3 \mathrm{D}^{\circledR}$, Inroads ${ }^{\circledR}$ e CLIP ${ }^{\circledR}$. Se conhecidos os valores para análise (a distância de visibilidade de parada e as características do motorista e do objeto), os programas verificam tridimensionalmente se a visibilidade mínima necessária é garantida ao longo de trecho da rodovia verificando as interseções da linha de visão do motorista com outros objetos do projeto ou do próprio terreno, simulados através de modelos digitais.

\section{METODOLOGIA}

O foco do estudo foi investigar o posicionamento de barreiras rígidas centrais para que não exista interferência na distância de visibilidade de parada necessária ao motorista durante o tráfego em determinada via, em trechos de conversão à esquerda, onde a barreira tem maior probabilidade de obstruir a visibilidade, quando utilizada como separador físico. Aspectos inerentes às suas funções de segurança da barreira, como dimensionamento estrutural e tipo de material construtivo, foram desconsiderados. $\mathrm{O}$ posicionamento da barreira foi sintetizado pelo dimensionamento do refúgio - espaço entre a faixa de rolamento interna da via e a barreira rígida central - aqui denominado afastamento lateral da barreira, como ilustrado na Figura 1 .

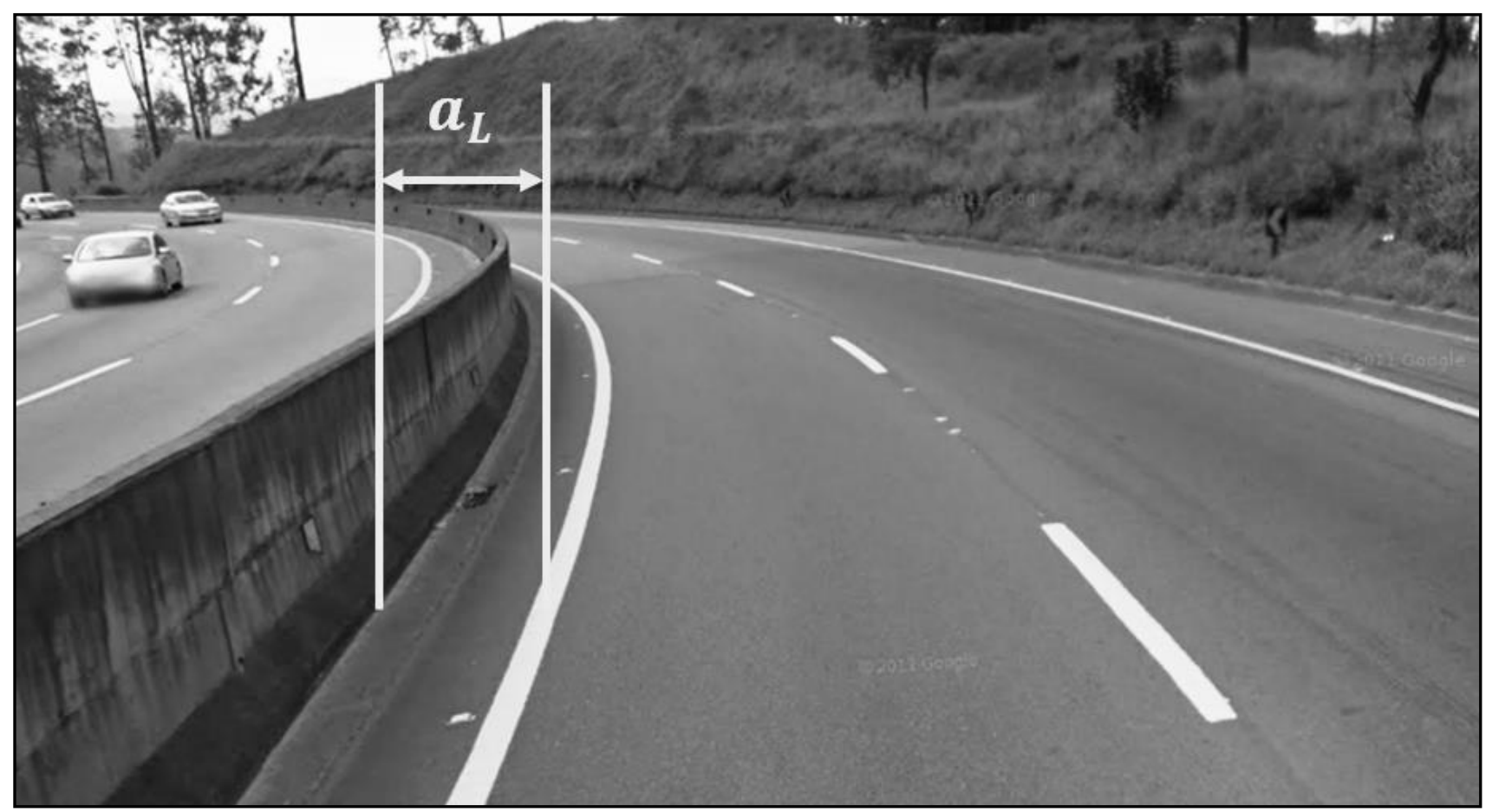

Figura 1 - Afastamento lateral da barreira

O artifício adotado foi o uso de modelos tridimensionais de projetos viários, em ambiente virtual, e sobre estes, interferiu-se na parametrização para a compreensão da relação da barreira central com a visibilidade existente na via, em virtude dos propósitos do estudo.

\subsection{Hipóteses Adotadas}

Em função da amplitude de parâmetros, foram adotadas hipóteses e simplificações nos projetos geométricos analisados no intuito de restringir abrangência do universo de estudo. Em síntese, as hipóteses adotadas foram:

- Rodovias de estudo: rodovias bidirecionais em concordância com as classes 0 e I-A definidas pelo DNER (1999).

- Velocidades de Projeto: Valores de velocidade de projeto e de DVP em concordância com a Tabela 2, calculados pela formulação apresentada na equação (1), segundo DER-SP (2006), considerando o tempo de percepção e reação igual a 2,5 s e a taxa de desaceleração de $3,4 \mathrm{~m} / \mathrm{s}^{2}$. 
Tabela 2 - Valores Mínimos de Projeto para DVP

\begin{tabular}{c|ccccccc}
\hline \multicolumn{7}{|c}{ Valores Mínimos de Projeto para DVP $(\mathrm{m})$} \\
\hline $\begin{array}{c}\text { Velocidade } \\
(\mathrm{km} / \mathrm{h})\end{array}$ & $-9 \%$ & $-6 \%$ & $-3 \%$ & $0 \%$ & $3 \%$ & $6 \%$ & $9 \%$ \\
\hline 60 & 97 & 92 & 87 & 85 & 80 & 77 & 75 \\
70 & 124 & 116 & 110 & 105 & 100 & 97 & 93 \\
80 & 154 & 144 & 136 & 130 & 123 & 118 & 114 \\
90 & 187 & 174 & 164 & 160 & 148 & 141 & 136 \\
100 & 223 & 207 & 194 & 185 & 174 & 167 & 160 \\
110 & 262 & 243 & 227 & 220 & 203 & 194 & 186 \\
120 & 304 & 281 & 263 & 250 & 234 & 223 & 214 \\
\hline
\end{tabular}

- Geometria: Curvas horizontais à esquerda, de raio variável entre $200 \mathrm{~m}$ e $2000 \mathrm{~m}$, incrementados a cada $100 \mathrm{~m}$. O limite superior de raio igual a $2000 \mathrm{~m}$ foi escolhido pelo fato que o problema de visibilidade em curvas horizontais à esquerda é pouco recorrente quando se assume grandes raios horizontais. Os limites inferiores, por sua vez, coincidem com o raio mínimo da curva horizontal para cada velocidade de projeto. As curvas são desenvolvidas em greide de declividade constante. Tal combinação é utilizada para compensar grandes desníveis de altitude, como casos de trechos de rodovias em regiões montanhosas. Os valores deste parâmetro variaram de $-9 \%$ a $9 \%$, incorporando aclives, declives e áreas planas.

- Seção transversal: Pista dupla, em ambos os sentidos, divididas por barreira rígida e afastamento, este variável (pois se trata do valor estudado) em relação à borda interna da pista de rolamento. Em casos que o raio da curva horizontal seja superior ao raio mínimo, é necessário dimensionar a superelevação. Adotou-se a redução gradual dos valores de superelevação, em função do aumento do raio, até atingir valor mínimo admissível, igual a $2 \%$ visando facilitar a drenagem das águas pluviais sobre a via. A superelevação máxima admissível neste estudo foi igual a $8 \%$, compatível com os padrões de rodovias escolhidos.

- Barreiras: barreiras rígidas duplas com perfil New Jersey, de alturas iguais a $0,81 \mathrm{~m}, 1,00 \mathrm{~m} \mathrm{e}$ $1,40 \mathrm{~m}$.

- Posicionamento e altura dos olhos do motorista e do objeto: Serão considerados os valores de 1,08 $\mathrm{m}$ para altura dos olhos do motorista e de $0,60 \mathrm{~m}$ para a altura do objeto a ser visualizado na via neste estudo (DNIT, 2010; DER-SP, 2006; AASHTO, 2011a). A altura do objeto adotada representa a posição média das lanternas traseiras de um veículo de passeio, que consistem no obstáculo com maior ocorrência de acidentes graves nas rodovias, quando na ausência de iluminação da via. Valores inferiores a 0,60 m, para AASTHO (2011a), poderiam aumentar os custos de implantação da estrada, já que o traçado deveria ser adequado, além de que haveria dificuldades por parte do motorista em detectar objetos desta dimensão sobre a via. A posição tanto do motorista quanto do objeto é de $2 \mathrm{~m}$ da borda direita da faixa de rolamento interna da via.

\subsection{Estruturação e execução das simulações}

As simulações foram feitas no AutoCAD ${ }^{\circledR}$ Civil3D ${ }^{\circledR}$. Inicialmente criou-se um modelo digital de terreno hipotético, através de um conjunto de pontos cotados, necessário para o processo de simulação no programa. Nas análises, contudo, a informação do terreno foi desprezada, já que os fatores considerados como obstrução foram oriundos apenas de elementos do projeto geométrico, sem incorporar taludes e outras modificações no relevo. Em relação ao traçado, os trechos de projeto elaborados possuem extensão média de $3 \mathrm{~km}$, abrangendo duas tangentes fixas. As curvas de análise foram adequadas a estas duas tangentes, sem a adição de curvas de transição. Adotou-se um sistema métrico arbitrário de coordenadas e orientação, já que o interesse estava na posição relativa entre as feições no projeto viário.

O ângulo central das curvas foi de $90^{\circ}$ para qualquer raio analisado. Previamente, ângulos centrais distintos, maiores e menores que $90^{\circ}$, foram testados. Não foram constatadas diferenças significativas, ou seja, superior à precisão de 0,05 $\mathrm{m}$ estipulada para a execução das simulações. $\mathrm{O}$ fato que nos demais casos testados o desenvolvimento em curva sempre foi maior do que a DVP analisada, não interferindo na acomodação da DVP sobre a via, durante a análise de visibilidade. Situações em que a DVP seja maior que o desenvolvimento da curva devem ser analisadas individualmente.

Em seguida, definiu-se cenários de análise em função da altura da barreira e da velocidade de projeto. Para cada altura de barreira considerada, 
sete cenários foram simulados referentes às velocidades de $60 \mathrm{~km} / \mathrm{h}$ a $120 \mathrm{~km} / \mathrm{h}$. Nos cenários configurados verificou-se para cada raio da curva horizontal se a DVP referente à velocidade e à declividade do greide é satisfeita. Caso não seja satisfeita, o posicionamento da barreira na seção transversal é modificado (afastamento lateral) até que se obtenha resposta positiva. $\mathrm{O}$ processo termina quando todo o conjunto de cenários for testado.

O valor máximo de afastamento lateral considerado foi de $2,50 \mathrm{~m}$, valor mediano aos encontrados na literatura. Além disso, adotou-se como critério de precisão do afastamento da barreira sendo igual $\mathrm{a} \pm 0,05 \mathrm{~m}$, valor este satisfatório ao considerar a precisão construtiva de uma rodovia. A visibilidade foi analisada a cada $10 \mathrm{~m}$ ao longo do trecho de estudo.

\section{ANÁLISE DOS RESULTADOS DAS SIMULAÇÕES}

As simulações realizadas considerando as hipóteses apresentadas e barreiras de 0,81 m de altura retornaram para todos os casos afastamentos laterais da barreira nulos. Isto não significa ausências de problemas de obstrução com barreiras desta altura, mas sim que a metodologia proposta foi incapaz de modelar os fatores que podem interferir na análise de visibilidade.

Uma questão a ser investigada é se caso a consideração de coordenação com curvas verticais tivesse sido uma das hipóteses premissas deste estudo, ao invés de rampas de greide constante, o resultado seria mais próximo do real. Para tal, cenários foram criados com curvas à esquerda englobando as hipóteses anteriores e a ocorrência de curvas verticais - côncavas e convexas. As distâncias de visibilidade foram testadas e os afastamentos laterais definidos. Em curvas côncavas, os afastamentos laterais da barreira mínimos continuaram nulos para a garantia da DVP. Já em curvas convexas, problemas de visibilidade foram constatados, indicando uma limitação da metodologia proposta.

Assim sendo, apenas os dados das barreiras de altura de $1,00 \mathrm{~m} \mathrm{e} 1,40 \mathrm{~m}$ foram tratados neste trabalho, definindo-se dois grupos de análise denominados de A e B. O grupo A compreendeu as simulações referentes à barreira de altura igual a $1,00 \mathrm{~m}$, com sete cenários de análise definidos pelas velocidades de projeto analisadas. As simulações do grupo A totalizaram 586 valores de afastamento lateral da barreira. Da mesma forma, o grupo B abrangeu as simulações referentes à barreira de altura igual a $1,40 \mathrm{~m}$, com cenários similares ao do grupo anterior A, totalizando 335 valores de afastamento lateral.

Para o grupo B, que engloba observações de barreiras de 1,40 m, os resultados das simulações foram organizados na forma de diagramas, um para cada velocidade de projeto considerada. Nos diagramas, à medida que o raio da curva horizontal diminui exige-se um afastamento lateral de dimensão maior para que seja garantida a DVP em toda a extensão do projeto analisado. A redução ocorre até um ponto onde o afastamento necessário se iguala ao máximo tolerável de $2,50 \mathrm{~m}$, sendo que os raios inferiores a essa situação foram desprezados.

Os diagramas gerados são coerentes com a formulação adotada, à medida que a declividade do greide aumenta, os valores de DVP diminuem. $\mathrm{O}$ resultado visual é que para cada velocidade, as curvas de declividades são dispostas lado a lado, sem sobreposição. Logo é possível identificar nos diagramas combinações onde a DVP será satisfeita, que são as áreas descritas acima de cada curva de declividade, como exemplificado na Figura 2.

$\mathrm{Na}$ parte superior da Figura 2, a área em cinza claro em destaque mostra as combinações possíveis para que a DVP igual a $148 \mathrm{~m}$ seja satisfeita, que se refere à velocidade de $90 \mathrm{~km} / \mathrm{h}$ e declividade do greide de $3 \%$. Analogamente, na parte inferior da Figura 2, a área em cinza escuro destacada indica as combinações válidas para a DVP igual a $187 \mathrm{~m}$, referente à mesma velocidade com declividade do greide igual a $-9 \%$. A análise é válida para os demais diagramas do grupo B.

Em contrapartida, os diagramas gerados com as observações das simulações do grupo A, exemplificados na Figura 3, tiveram comportamento distinto daqueles oriundos do grupo B. À medida que o raio da curva horizontal diminui, o afastamento lateral da barreira aumenta até certo ponto em que ocorre inversão deste comportamento, com diminuição dos afastamentos até atingir o raio mínimo de análise.

Como exemplo apresenta-se a Figura 3. Na parte superior, a área em cinza claro em destaque mostra as combinações possíveis para que a DVP igual a $148 \mathrm{~m}$ seja satisfeita, que se refere à velocidade de $90 \mathrm{~km} / \mathrm{h}$ e declividade do greide de $3 \%$. Usando o mesmo diagrama, na parte inferior, a área em cinza escuro indica as combinações válidas para a DVP igual $187 \mathrm{~m}$, referente à mesma 
velocidade com declividade do greide de $-9 \%$. A análise é válida para os demais diagramas do grupo A.

O esperado seria comportamento similar ao do grupo B. Contudo com a análise tridimensional dos cenários identificou-se que parâmetros de projeto, como superelevação e declividade do greide, contribuem na posição espacial dos elementos de análise - motorista, objeto e barreira - de modo a favorecer a visibilidade de projeto. A barreira com altura de 1,00 m torna-se uma obstrução não significativa à visibilidade e pode ser compensada por algumas variações na parametrização do projeto. Por outro lado, a barreira do grupo B com altura de $1,40 \mathrm{~m}$, por suas dimensões, acaba sendo uma obstrução intransponível à visibilidade.

A Figura 4 apresenta duas cenas de um dos modelos tridimensionais do grupo A, com representações do observador - o motorista - e do objeto na pista que implica na parada do veículo.
Ambos estão posicionados conforme as hipóteses da simulação. O segmento em destaque é a linha de visão do motorista, que no caso I, transpõe a barreira sem nenhuma obstrução. Em II é imposta uma leve diminuição do afastamento lateral da barreira, obstruindo a visibilidade do motorista, gerando uma zona oclusa aos seus olhos (segmento preto) na qual se encontra o objeto a ser observado, de modo que o critério de DVP não é satisfeito. Nota-se que, para cenários do grupo A, a visibilidade do objeto ocorre sobre a barreira, auxiliado pelo afastamento lateral imposto. Em testes realizados, constatou-se que o mesmo comportamento ocorre para barreiras de altura de $0,81 \mathrm{~m}$.

Por fim, os diagramas gerados do grupo A podem ser analisados da mesma forma que os do grupo B, onde áreas acima das curvas de declividade do greide representam combinações onde a DVP é garantida.
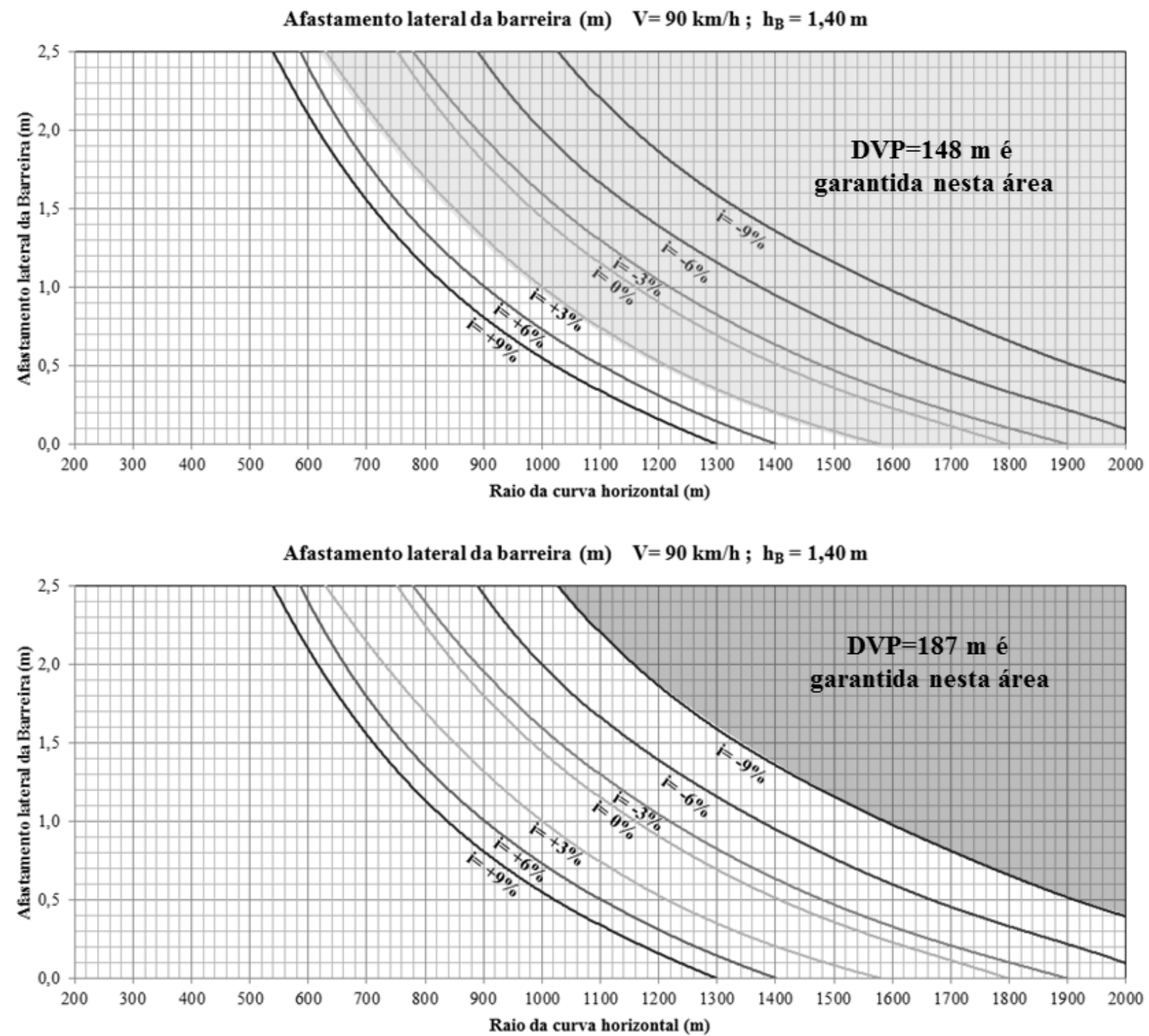

Figura 2 - Delimitação de regiões de combinações válidas para a garantia da DVP analisada - Grupo B 


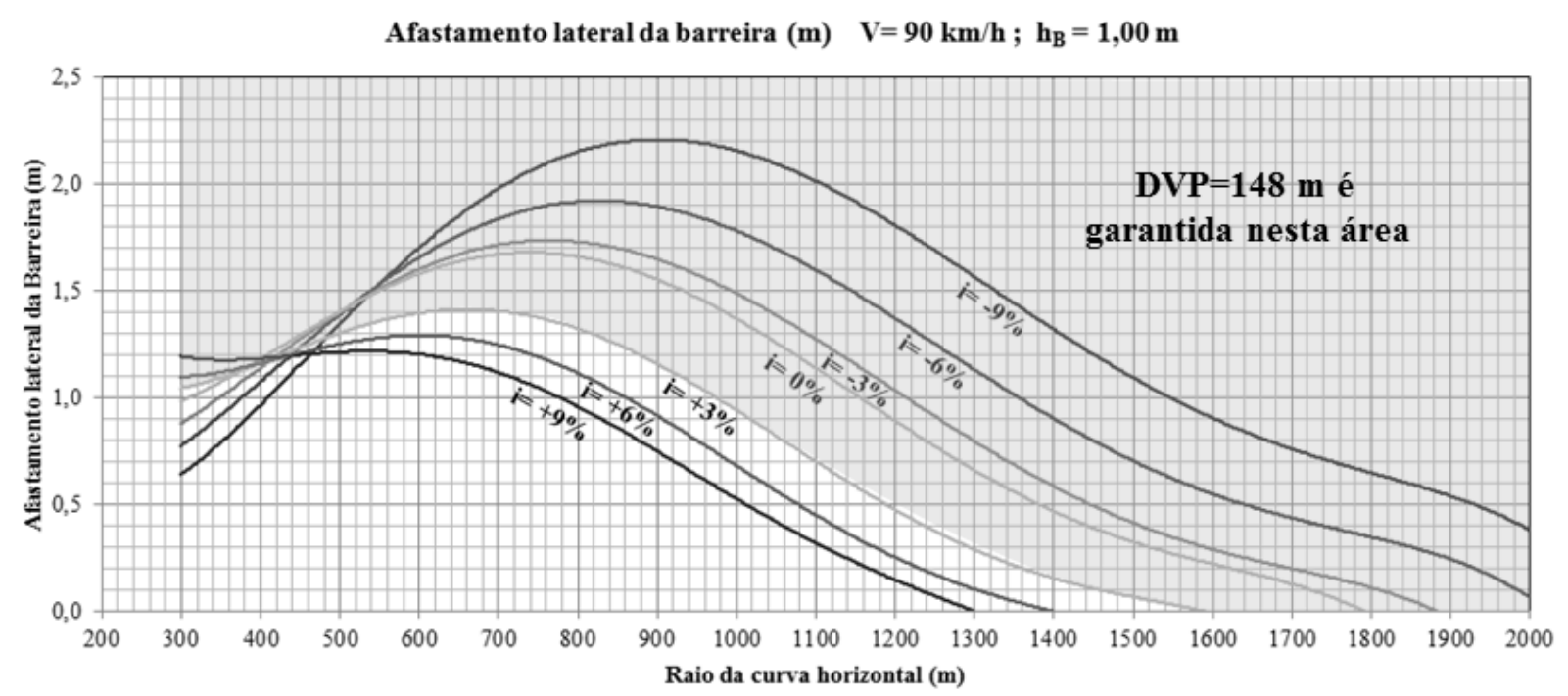

Afastamento lateral da barreira (m) $V=90 \mathrm{~km} / \mathrm{h} ; \mathrm{h}_{\mathrm{B}}=1,00 \mathrm{~m}$

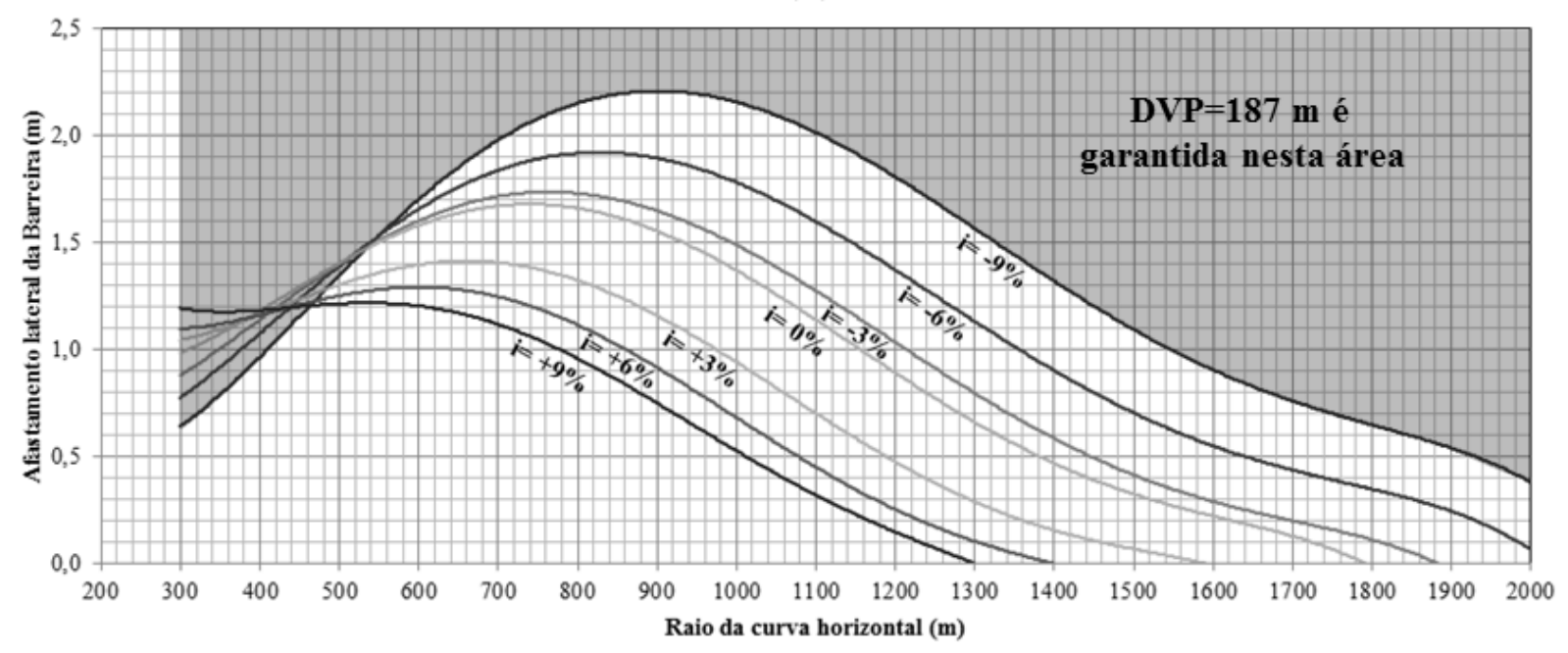

Figura 3 - Delimitação de regiões de combinações válidas para a garantia da DVP analisada - Grupo A

\section{CONCLUSÕES E RECOMENDAÇÕES}

As condições de visibilidade provêm da composição dos alinhamentos horizontal, vertical e da seção transversal e, portanto, qualquer alteração na geometria da via é capaz de modificar a visibilidade existente. Nas simulações realizadas foi possível a análise da problemática - posicionamento de barreiras e visibilidade - considerando a influência dos elementos de projeto em conjunto.

Os dados simulados com barreira de altura de 0,81 m retornaram para todas as hipóteses afastamento necessário nulo. Todavia, isso não significa que não ocorram problemas de visibilidade com barreiras de $0,81 \mathrm{~m}$. O fato é que as hipóteses adotadas não foram capazes de modelar todas as variáveis envolvidas na análise de visibilidade, tornando a metodologia inadequada para análises de barreiras ditas de altura baixa. Nos demais ca- sos, barreiras de altura de $1,00 \mathrm{~m}$ e $1,40 \mathrm{~m}$, a metodologia proposta mostrou-se capaz de modelar as variáveis que interferem na análise de visibilidade, apesar das limitações das hipóteses adotadas. Os diagramas do conjunto B (altura da barreira de $1,40 \mathrm{~m}$ ) tiveram comportamento próximo ao esperado. A visibilidade foi definida por afastamentos mínimos que permitissem a visualização do objeto, a uma dada DVP, com visada tangenciando a barreira central. Dessa forma, à medida que o raio da curva horizontal diminui, o afastamento entre barreira e a faixa de rolamento aumenta, de modo a garantir a manutenção da DVP. Trechos em aclives atenuam os afastamentos laterais da barreira, de modo que curvas de declividades diferentes se apresentam levemente deslocadas para a mesma velocidade de projeto analisada. 

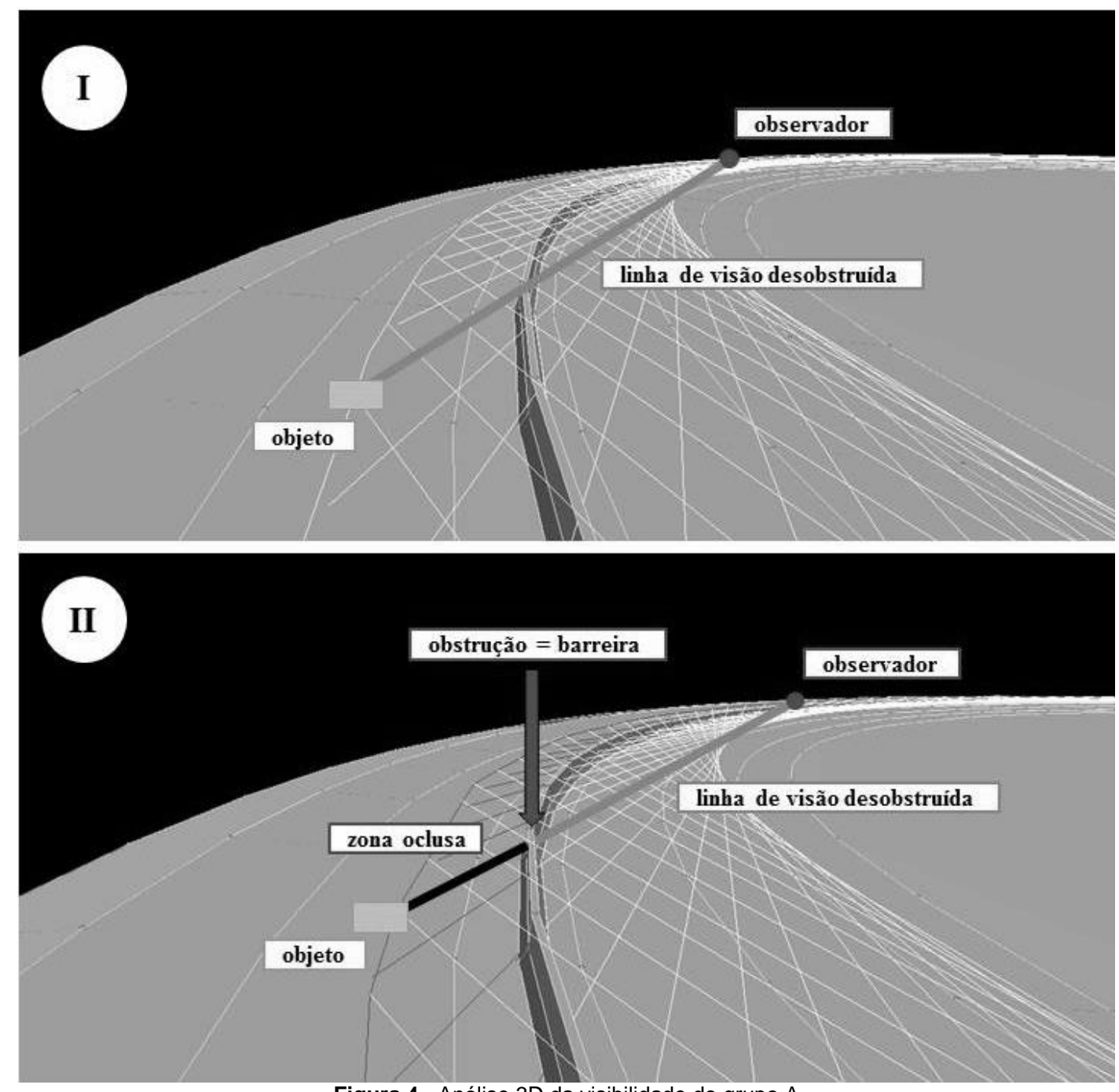

Figura 4 - Análise 3D da visibilidade do grupo A

Todavia, para o grupo A (altura da barreira de $1,00 \mathrm{~m}$ ) os diagramas apresentaram comportamento distinto. À medida que o raio da curva horizontal diminui, o afastamento lateral da barreira alcança um valor máximo. Deste ponto, há um decréscimo do afastamento em função da diminuição do raio da curva horizontal. O motivo é que a solução mínima fornecida pelo programa durante a simulação é de sempre visualizar o objeto sobre a barreira, transpondo-a com uma visada inclinada.

As simulações computacionais tridimensionais mostraram-se uma forma promissora e de baixo custo para investigar a influência de objetos e de características do projeto nos padrões de segurança da via, principalmente durante a fase de estudo e planejamento do traçado. A facilidade de alterar valores de parâmetros de projeto e, testar a visibilidade considerando o projeto como o todo, torna as análises mais realistas. É possível gerar pontos de vista diferentes e, de tal forma, melhor compreender problemas de obstrução na via, que dificilmente seriam identificados utilizando as análises bidimensionais, com alinhamentos horizontal e vertical tratados separadamente. Porém, o conjunto de parâmetros variáveis foi limitado pelo tempo gasto para a criação e execução das simulações. Em geral, para cada valor encontrado no estudo, pelo menos outros três afastamentos (maiores e menores) foram testados de forma a validar o valor encontrado, dentro da precisão considerada de $\pm 0,05 \mathrm{~m}$. Por isso, o volume de análises e esforços foi superior ao estimado inicialmente. Essa desvantagem pode ser mitigada ao empregar rotinas computacionais para a geração dos modelos e testes de visibilidade de forma automática, que minimizam a interferência humana além de acelerar o processo de geração de dados.

Por fim, ressalta-se que os afastamentos definidos serão recomendações iniciais para o posicionamento de barreiras quando considerada a garantia da DVP. Para o estabelecimento de padrões mais consistentes recomenda-se para trabalhos fu- 
turos ampliar o conjunto de hipóteses incluindo projetos viários mais completos do que os simulados, agregando a coordenação entre os alinhamentos horizontal e vertical, adoção de modelos matemáticos multivariados, visando melhor representar o comportamento dos dados, bem como investigar a influência de cada variável na determinação do afastamento lateral da barreira.

\section{AGRADECIMENTOS}

Os autores agradecem a CAPES (Coordenação de Aperfeiçoamento de Pessoal de Nível Superior), ao CNPq (Conselho Nacional de Desenvolvimento Científico e Tecnológico) e ao Departamento de Engenharia de Transportes (PTR) da Escola Politécnica da Universidade de São Paulo (EPUSP) pelos recursos materiais e financeiros disponibilizados.

\section{REFERÊNCIAS}

AASTHO (2011a) A Policy on Geometric Design of Highways and Streets. 6 ed. American Association of State Highway and Transportation Officials. Washington D.C.

AASTHO (2011b) Roadside Design Guide. 4 ed. American Association of State Highway and Transportation Officials. Washington D.C.

ABNT (2007). NBR 15486 - Segurança no tráfego - Dispositivos de contenção viária - Diretrizes. Associação Brasileira de Normas Técnicas, Rio de Janeiro.

Arndt, O. K.; Cox, R. L.; Lennie, S. C.; Whitehead, M. T. (2010) Provision of Sight Distance around Concrete Barriers and Structures on Freeways and Interchanges. $4^{\text {th }}$ International Symposium on Highway Geometric Design. Valencia.

AUSTROADS (2010). Guide to Road Design - Part 3: Geometric Design. 2 ed. Sidney.

Chen, E.; Brown, J.; Tarko, A. (2012) Use of barriers in rural open road conditions - a synthesis study. JTRP Technical Reports - Joint Transportation Resarch Program. Indiana.

Chou, A. T. M.; Pérez, V. M. F.; García, A. G.; Rojas, M. A. R. (2010) Optimal 3D Coordination to Maximize the Available Stopping Sight Distance in Two-lane Roads. $4^{\text {th }}$ International Symposium on Highway Geometric Design. Valencia.

DER-SP (2006) Notas Técnicas de Projeto Geométrico. Departamento de Estradas de Rodagem do Estado de São Paulo, Diretoria de Engenharia. São Paulo.

DNER (1999) Manual de Projeto Geométrico de Rodovias Rurais. Departamento Nacional de Estradas de Rodagem, Diretoria de Desenvolvimento Tecnológico, Divisão de Capitação Tecnológica. Rio de Janeiro.
DNIT (2010) Manual de Projeto Geométrico de Vias Urbanas. Departamento Nacional de Infraestrutura de Transportes. Diretoria Executiva. Instituto de Pesquisas Rodoviárias. Rio de Janeiro.

Han, K.; Middleton, D.; Clayton, A. (2006) Enhancing Highway Geometric Design: Development of Interactive Virtual Reality Visualization with Open-Source Technologies. Journal of Transportation Research Board. n. 1980, p. 134-142.

Hassan, Y.; Easa, S. M.; Abd El Halim, A. O. (1997) Design Considerations for Combined Highway Alignments. Journal of Transportation Engineering. v. 123. n. 1, p. 60-68.

Hassan, Y.; Sarhan, M. (2012) Consideration of Sight Distance in Placement of Concrete Barriers on Horizontal Curves of Roads. Journal of Transportation Research Board. n. 2301, p. 9-16.

Hixon III, C. L. (2007) Visualization Issues for Transportation Agencies - Approaches and Challenges. Transportation Research News. n.252, p. 11-14.

Ismail, K.; Sayed, T. (2007) New Algorithm for Calculating 3D Available Sight Distance. Journal of Transportation Engineering. v. 133 . n. 10 , p. $572-581$.

Janikula, T.; Garrick, N. W. (2002) Three-Dimensional Visualization Approach to Illustrating Esthetic Concepts for Highway Design. Transportation Research Record. n. 1796, p. 3540

Kuhn, W.; Jha, M. K. (2010) Methodology for Checking Shortcomings in the Three-Dimensional Alignment. $4^{\text {th }}$ International Symposium on Highway Geometric Design. Valencia.

Leisch, J. P. (1989) Horizontal Sight Distance Considerations in Freeway and Interchange Reconstruction. Transportation Research Record - Highway Sight Design Issues. n. 1208. Washington D.C, p. $80-84$.

NCHRP. (2006) NCHRP Synthesis - Visualization for Project Development: A Synthesis of Highway Practice. National Cooperative Highway Research Program. Washington D. C.

Neuman, T. R. (1989) New Approach to Design for Stopping Sight Distance. Transportation Research Record - Highway Sight Design Issues. n. 1208. Washington D.C., p. 14-22.

Pellegrini, P. T. (2006) Contribuição para o Estudo das Distâncias de Visibilidade de Ultrapassagem para Rodovias Bidirecionais com Duas Faixas de Tráfego. Dissertação de Mestrado. São Paulo: Universidade de São Paulo.

Rosey, F.; Auberlet, J. M. (2012) Trajectory variability: Road geometru difficult indicator. Safety Science. v 50. n. 9, p. 18181828.

Sanchez, E. (1994) Three-Dimensional Analysis of Sight Distance on Interchange Connectors. Transportation Research Record Highway and Facility Design Cross-section and Alinement Design Issues. n. 1445. Washington D.C., p. 101-108.

Yang, Q.; Overton, R.; Han, L. D.; Yan, X.; Richards, S. H. (2012) The influence of curbs on driver behaviors in four-lane rural highways - A driving simulator based study. Accident Analysis and Prevention. v. 50, p.1289-1297. 